\title{
3.6 CRAB PULSAR RADIATION CHARACTERISTICS
}

\author{
R. N. MANCHESTER \\ National Radio Astronomy Observatory*, Green Bank, W. V., U.S.A.
}

It has been suggested that for the Crab pulsar the radio radiation mechanism may be different from that in other pulsars. The principal observations leading to this suggestion are, firstly, the essentially constant position angle of the highly linearly polarised precursor, and secondly, the occasional large increases in intensity of the main pulse.

I would like briefly to point out that similar characteristics have been observed in other pulsars, and therefore to suggest that the Crab pulsar is not unique in this respect. Highly polarised precursor pulses have been observed in a number of pulsars, particularly PSR $0329+54$ and PSR $0950+08$. In each case the variation of position angle through this precursor pulse is small.

An interesting phenomenon has been observed in PSR $1237+25$ which may be related to the erratic amplitude behaviour of the Crab pulsar main pulse. The mean pulse profile for this pulsar is shown in the lower part of Figure 1. It is clear that there are at least five components making up the average profile. Occasionally however the central component becomes much more intense, generally dominating the pulse profile. This strong central pulse in PSR $1237+25$ is narrow and only weakly polarised - similar to the Crab pulsar main pulse. The variation of position angle shown in the upper portion of Figure 1 suggests that we are looking directly down on a magnetic pole for this pulsar. If this interpretation is accepted, then the erratic central pulse is being emitted directly from the pole. These results suggest that for the Crab pulsar the line of sight may pass through a magnetic pole as for PSR $1237+25$, with the main pulse being emitted from the pole.

\section{Discussion}

C. Heiles: Many pulsars have components which vary relative to each other by large factors. The Crab pulsar is qualitatively different, however; the main pulse occasionally becomes a factor of fully 2000 times the average pulse density.

$R$. N. Manchester: The erratic centre pulse from PSR 1237 can be up to about a 100 times as strong as the mean.

F. C. Michel: There appears to be a systematic tendency for the sweep in polarisation to occur when the total polarisation or intensity is small - is this a general feature?

$R$. N. Manchester: The polarised component is strong at the position of the P.A. swing in PSR 2045, and so the swing cannot be accounted for by a drop in the polarised power. For PSR 1237 the polarised component is low at the position of the swing in position angle. However, the P.A. of the two sides of the pulse is almost the same, so again the swing cannot be explained by this.

* Operated by Associated Universities, Inc., under contract with the National Science Foundation. 


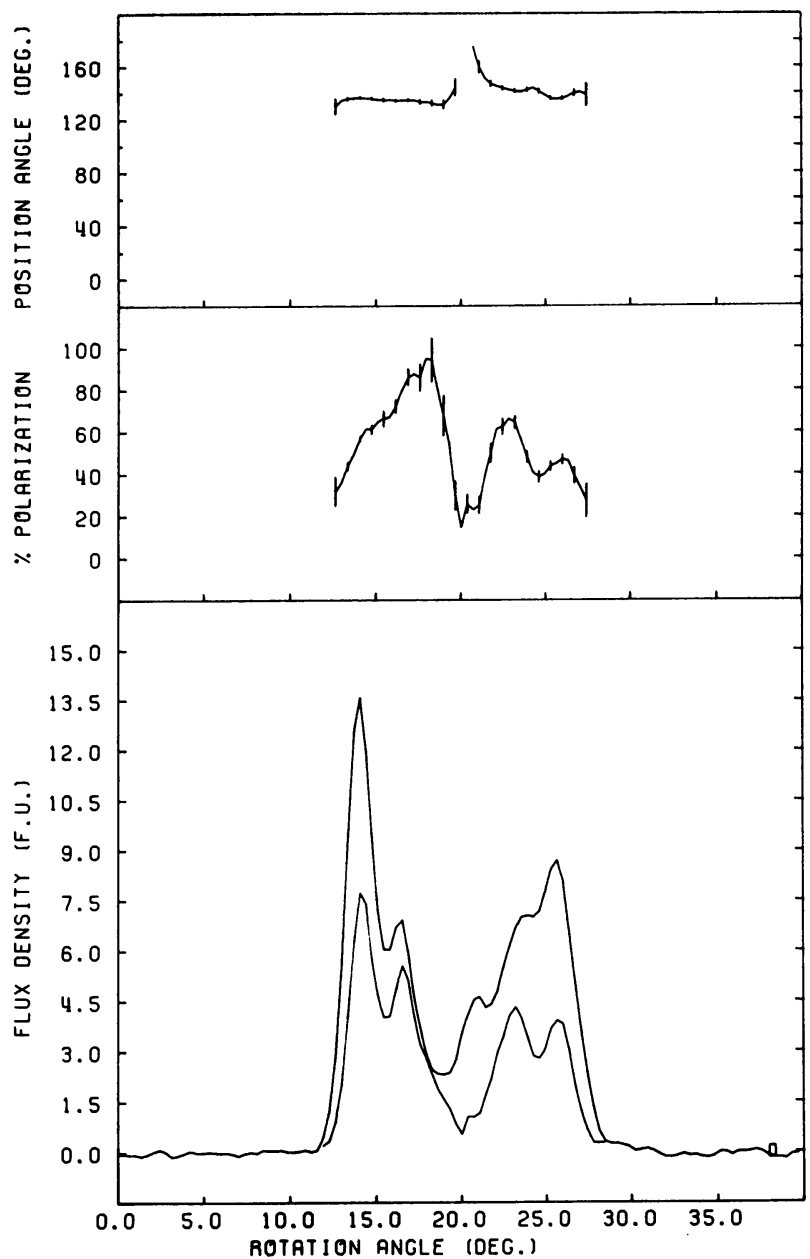

Fig. 1. Mean pulse profile for PSR $1237+25$ measured at $410 \mathrm{MHz}$. The lower portion of the figure gives the total intensity and linearly polarised component, the central portion, the percentage linear polarisation, and the upper portion, the position angle of the linear component. 